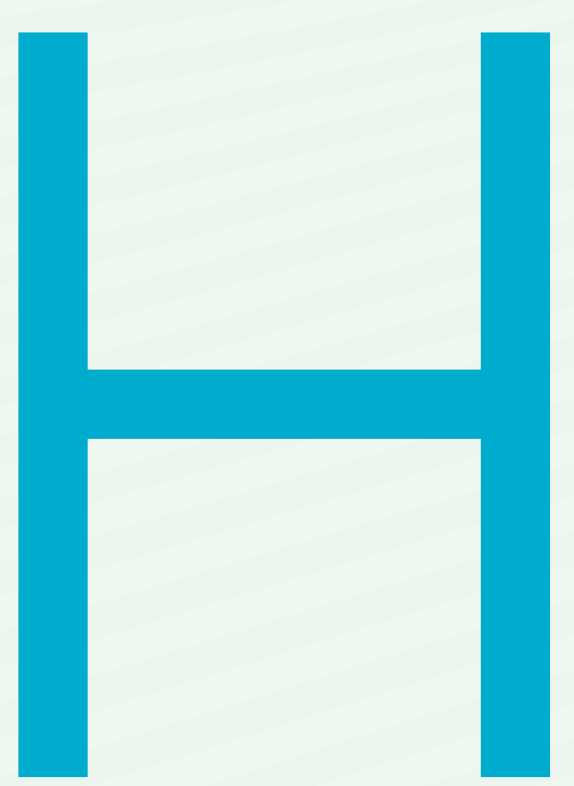

\title{
The NII Shonan Meeting in Japan
}

BY KEN-ICHI KAWARABAYASHI

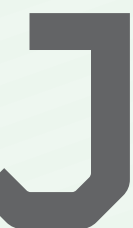

APAN'S NATIONAL INSTITUTE of Informatics (NII) launched its inaugural NII Shonan Meeting in February 2011. It was the first international conference of informatics in Asia, following in the style of the Dagstuhl seminars in Germany, designed to bring together the world's leading researchers and engineers to discuss open problems and challenges. More than 140 meetings have been held since then, and the number of participants totaled approxi- mately 3,500 by November 2019. NII supports all the administrative arrangements for organizers and covers approximately half the fee for every academic participant (including room, board, and meeting fees). We currently hold 20-25 workshops/year. Sometimes, we also have summer/winter schools.

A recent trend in computer science conferences is to host "mega" conferences. For example, both the NeurIPS and CVPR events have attracted thousands of participants. There are many workshops

\section{A typical NII Shonan event focuses on smaller but emerging areas of informatics with around 25 participants.}

co-located with these mega conferences, but because they usually last a day or less it is very difficult for participants to interact with each other. This prompts the research community to have smaller workshops that last at least a few days. Dagstuhl is the most successful of this kind and an excellent model for the NII Shonan Meeting.

A typical NII Shonan event focuses on smaller but emerging areas of informatics with around 25 participants. Each meeting invites experienced and young promising researchers in academia and industry for interdisciplinary discussions. As of November 2019, participants have represented 60 countries and regions: $38 \%$ from Asia, $37 \%$ from Europe, 20\% from North America and South America, $4 \%$ from Oceania, and less than $1 \%$ from Africa. In the last few years, about $13 \%$ of the participants are female researchers.
In such international surroundings, the NII Shonan Meeting encourages close communication among participants. The venue - Shonan Village Center-offers a conducive environment for such gatherings, located far from downtown on a hilltop with lush greenery. Each meeting lasts 4-5 days, including a half-day excursion. By the end of every workshop, each participant has learned the research interests and expertise of all fellow attendees through days of discussions and friendly interactions.

To hold a Shonan Meeting, organizers must submit a proposal to the NII's academic committee. Experts exam the documents and organizers are notified about the results. Although similar to the Dagstuhl's review process, we often request proposal revisions, taking reviewers' comments into account. Sometimes, organizers are 


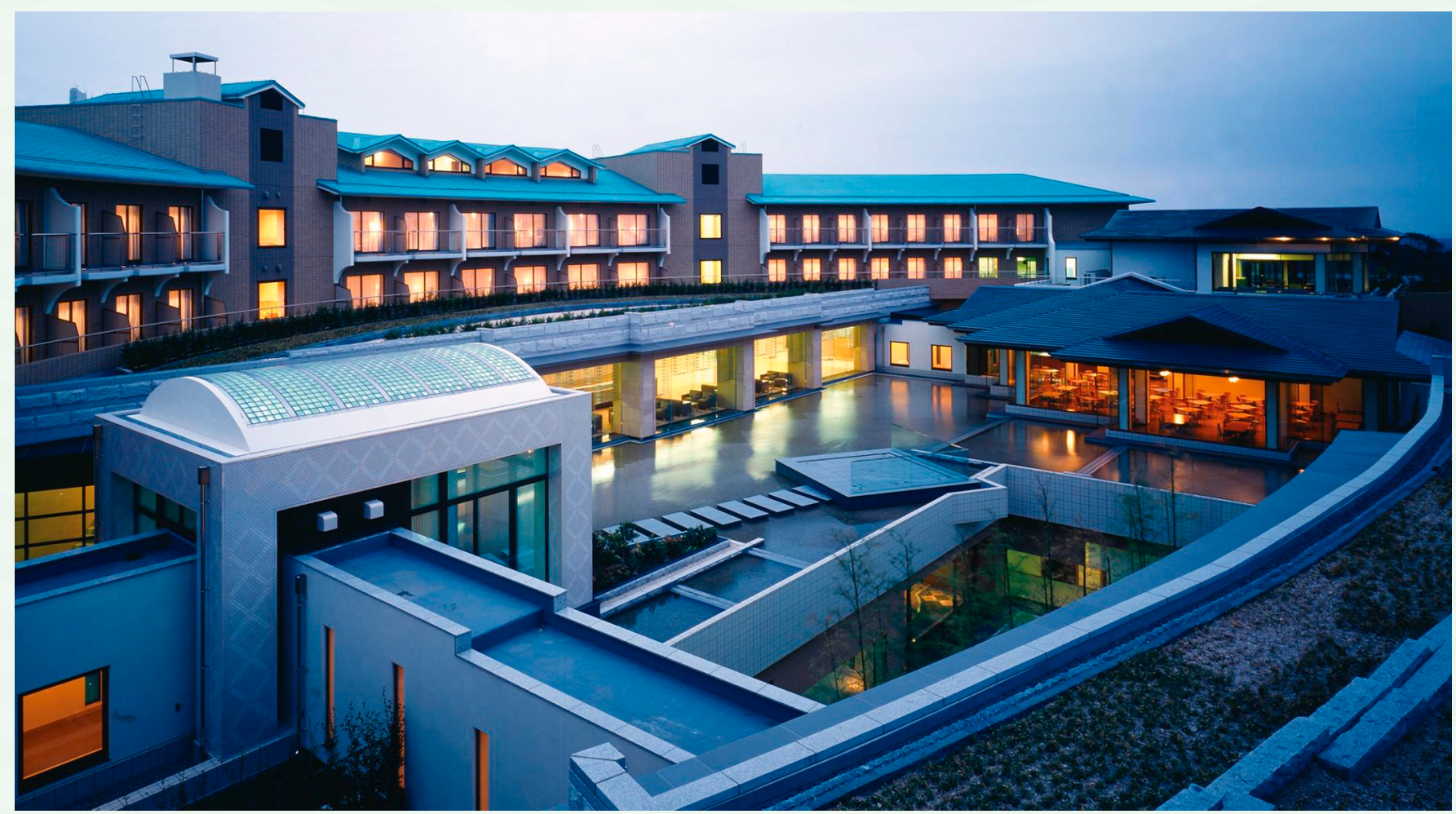

Every NII Shonan Meeting takes place at the scenic Shonan Village Center in Japan.

asked to make major changes to their proposals; in fact, on occasion they are asked for multiple revisions.

The computing areas covered by the NII Shonan Meet are very diverse. Indeed, in the last two years, software, theory, programming languages, and architecture (in a broad sense) have been the major focus, but they each occupy at most $20 \%$. There are several workshops in the area of natural language processing, machine learning, databases, and security. This is a bit different from the Dagstuhl model in that approximately $30 \%$ of those workshops focus on theory (in a broad sense), followed by programming languages (with around 15\%). Moreover, a Dagstuhl seminar typically hosts 40 researchers, while a NII Shonan Meeting: hosts 25 researchers on average. It appears the NII Shonan Meeting focuses on more specific areas while Dagstuhl's scope seems broader.

Remarkably, an increasing number of frequent participants have been creating research communities centered on their particular research topics. One example is a community on visualization formed after the No.046 seminar, ${ }^{a}$ that now organizes new workshops almost every year. Another group on engineering adaptive systems (EASy) has also been reuniting after No.004. ${ }^{\mathrm{b}}$

An important aspect of the NII Shonan Meeting is that collaborations have enabled researchers to publish books and papers and to obtain research grants. Researchers from No. $120^{\mathrm{c}}$ issued a paper on

a No.046; https://shonan.nii.ac.jp/ seminars/046/

b No.004; https://shonan.nii.ac.jp/ seminars/004/

c No.120; https://shonan.nii.ac.jp/ seminars/120/

\section{An important aspect of the NII Shonan Meeting is that collaborations have enabled researchers to publish books and papers and to obtain research grants.}

visual analytics and another group from No.074 $4^{\mathrm{d}}$ published a book on immersive analytics. Springer has published three monographs as a book series on Shonan workshops written by participants, one of which was written by EASy researchers. In addition, some researchers received research funding from NSF, SNSF, and JSPS, submitting proposals discussed during the

d No.074; https://shonan.nii.ac.jp seminars/074/

\section{Shonan seminars.}

Thus, the NII Shonan Meeting has contributed to research achievements by giving participants the opportunity to access broad ideas on specific topics. One of the missions of the NII Shonan Meeting is to welcome more participants from various backgrounds to this significant destination for informatics researchers. c

Ken-ichi Kawarabayashi is a professor and Deputy Director General at the National Institute of Informatics in Tokyo, Japan.

(C) 2020 ACM $0001-0782 / 20 / 4 \$ 15.00$ 